\title{
La idea de psicología racional en la Metafísica Alemana (1720) de Christian Wolff*
}

\author{
The Idea of Rational Psychology in Christian \\ Wolff's German Metaphysics (1720)
}

Recibido: enero 14 de 2014 | Revisado: julio 26 de 2014 | Aceptado: julio 27 de 2014

\author{
Saulo de Freitas Araujo** \\ Thiago Constâncio Ribeiro Pereira \\ Universidade Federal de Juiz de Fora, Minas Gerais, Brasil
}

doi:10.11144/Javeriana.upsy13-5.lipr

Para citar este artículo: Araujo, S. F., \& Pereira, T. C. R. (2014). La idea de psicología racional en la Metafísica Alemana (1720) de Christian Wolff. Universitas Psychologica, 13(5), 1655-1666. http:// dx.doi.org/10.11144/Javeriana.upsy13-5.lipr

Agradecimientos: Los autores agradecen al Consejo Nacional de Desarrollo Científico y Tecnológico (CNPq - Brasil), a la Coordenación de Perfeccionamento de Personal de Nivel Superior (CAPES - Brasil) y a la Universidade Federal de Juiz de Fora su apoyo financiero.

*** Correos electrónicos: saulo.araujo@ufjf.edu.br, thiagocrpereira@gmail.com

\section{RESUMEN}

Christian Wolff (1679-1754) fue una figura central en la Ilustración europea del siglo XVIII. Al mismo tiempo, tuvo una importancia particular para el desarrollo histórico de la psicología, pues dio a esta una nueva significación. Sin embargo, la historiografía tradicional de la psicología no le ha dado el debido reconocimiento. El objetivo de este artículo consiste en presentar un análisis teórico-conceptual de su psicología racional en su Metafísica Alemana (1720) y mostrar su importancia para los debates psicológicos posteriores. Con ello, se espera contribuir a la divulgación de un aspecto significativo del desarrollo histórico de la psicología.

Palabras clave

Christian Wolff; psicología racional; historia de la psicología

\section{A B S T R A C T}

Christian Wolff (1679-1754) was a central figure in the European Enlightenment in the $18^{\text {th }}$ century. At the same time, he played a special role in the historical development of psychology, since he gave it a new meaning. However, the historiography of psychology has not given him his due recognition. The aim of this paper is to offer a theoretical-conceptual analysis of his rational psychology in his German Metaphysics (1720) and to show its importance to subsequent psychological debates. Thus, we hope to contribute to the divulgation of an important aspect of the historical development of psychology.

Keywords

Christian Wolff; rational psychology; history of psychology 
El filósofo alemán Christian Wolff (1679-1754) es una figura central en la Ilustración europea del siglo XVIII y uno de los personajes más influyentes en la historia de la cultura alemana (Bossenbrook, 1961; École, 2001; González Ruiz, 2000; Pütz, 1978). Al mismo tiempo, tuvo una importancia particular para el desarrollo histórico de la psicología, pues dio a esta una nueva significación, es decir, proponiéndola como una ciencia especial con objeto, método, lenguaje y fronteras propias, aunque vinculada a un sistema filosófico más amplio (Blackwell, 1961; Corr, 1975; École, 1969; Gundlach, 2006). Así, el conocimiento psicológico adquirió contornos que lo separaban de la tradición aristotélica dominante, y le dieron un nuevo lugar como campo de investigación en el universo intelectual (Vidal, 2006).

Después de muchos años olvidada o superficialmente tratada en los estudios historiográficos, la psicología de Wolff ha despertado un nuevo interés en las dos últimas décadas, sea en relación con la historia de la psicología en el siglo XVIII (Araujo, 2012; Bell, 2005; Feuerhahn, 2002; Hatfield, 1995; Molina, 2010; Sturm, 2006; Vidal, 2006), sea por la búsqueda de una reinterpretación del pensamiento wolffiano (Marcolungo, 2007; Mei, 2011; Rudolph, \& Goubet, 2004). Sin embargo, estas nuevas investigaciones no han tenido todavía efecto sobre la historiografía tradicional de la psicología en general. En su mayoría, los más recientes manuales de historia de la disciplina siguen el hábito de los manuales antiguos (Baldwin, 1913; Boring, 1950; Brett, 1921; Watson, 1963), en los que la psicología wolffiana es completamente ignorada o, en el mejor de los casos, mencionada de manera superficial y equivocada (Goodwin, 2005; Hothersall, 2006; Robinson, 1995; Schultz, \& Schultz, 2011).

Pero incluso entre los estudios especializados en el pensamiento de Wolff existen insuficiencias. Por ejemplo, para hablar solo de la psicología, la mayor parte de ellos se concentra en los dos tratados latinos sobre el tema - Psychologia empirica (1732) y Psychologia rationalis (1734) -, dejando de lado la psicología desarrollada por Wolff en su Metafísica Alemana (MA). Siendo más extensos y más famosos que la MA, estos tratados latinos fueron tomados como la versión oficial de la psicología de Wolff.
Sin embargo, la MA no solo constituye la primera sistematización de la psicología de Wolff, sino también es una de las obras más populares e influyentes en el contexto intelectual alemán en aquella época (Corr, 2003; González Ruiz, 2000). Por eso, la carencia de investigaciones sobre la MA constituye una verdadera deficiencia en la literatura, independientemente de haber o no diferencias significativas entre ella y los textos de la fase latina de Wolff, una cuestión que no es posible investigar aquí.

En segundo lugar, muchos estudios están dedicados exclusivamente a su psicología empírica, devaluando su contraparte racional. Si bien Wolff propuso una separación entre la psicología empírica y la psicología racional, separación presentada por primera vez en la introducción general a sus tratados latinos-Discurso preliminar sobre la filosofía en general (1728)-, nos parece apresurada la afirmación de que solo la primera ha tenido una penetración real en el pensamiento psicológico del período (Vidal, 2006). Tal posición impide una comprensión adecuada de la concepción wolffiana de psicología en general. Además, distorsiona el valor de la psicología racional para el pensamiento wolffiano y para el desarrollo histórico de la psicología.

A partir de los problemas planteados, el objetivo de este artículo consiste en presentar un análisis teórico-conceptual de la psicología racional en la MA de Wolff, teniendo en cuenta los elementos esenciales no solo para la aprehensión de su contenido, sino también para la comprensión de su significación en el sistema wolffiano y en el contexto del siglo XVIII alemán ${ }^{1}$. Para eso, primeramente vamos a establecer su lugar en la obra inicial de Wolff. A continuación, presentaremos sus contenidos, contemplando su definición, sus temas centrales y algunas de sus relaciones conceptuales con otras disciplinas metafísicas. Finalmente, haremos algunas consideraciones acerca de su significado para el pensamiento wolffiano y para el curso posterior de

1 Aquí utilizaremos la $11^{\text {a }}$ edición alemana de la MA (1751), publicada en 2003 en la edición de las obras completas de Wolff (Christian Wolffs Gesalmmelte Werke), bajo la dirección de J.École. Para las citas, tomaremos como base la traducción española de González Ruiz (2000), haciendo pequeños cambios en caso necesario. 
la psicología. Con esto, esperamos contribuir a la divulgación de un aspecto importante del desarrollo histórico de la psicología.

\section{Filosofía y psicología en Wolff}

Es en el contexto de la Ilustración Alemana (Aufklärung) donde debemos empezar a comprender el proyecto psicológico de Wolff. Su formación intelectual fue marcada, en parte, por las controversias teológicas resultantes de la reforma protestante en Alemania, y en parte, por una admiración por el conocimiento matemático. A los 27 años de edad, Wolff se hizo profesor en la Universidad de Halle, después de una recomendación de G. W. Leibniz (1646-1716). Interesado en adquirir certeza matemática en los diversos campos del conocimiento humano y llegar a una fundamentación racional de la vida práctica, Wolff inició la elaboración de un sistema integral de filosofía, reuniendo y articulando elementos de diferentes tradiciones filosóficas (Hettche, 2009). De hecho, se nota allí la influencia de San Tomás de Aquino (1225-1274), R. Descartes (1596-1650), N. Malebranche (16381715), I. Newton (1643-1727), Leibniz, además de otros matemáticos, filósofos y científicos de su tiempo (Blackwell, 1961; González Ruiz, 2000). Por lo tanto, a pesar de su fuerte herencia escolástica, revelada principalmente en su estilo y su método de investigación, el emprendimiento de Wolff es un verdadero ejemplo de la búsqueda iluminista de un sistema unificado de conocimiento, de la libertad de pensamiento contra el control religioso y político, y de la fundamentación racional de una vida práctica virtuosa entre los hombres (École, 2001; Schwaiger, 2000).

Bajo la condición de Aufklärer, Wolff escribió sus primeras obras en alemán. De acuerdo con su extenso proyecto intelectual, elaboró, más allá de la MA (1720), una Lógica (1713), una Ética (1720), una Política (1721), una Física (1723), una Teleología (1724) y una Fisiología (1725). Todas estas obras fueron ampliamente conocidas en la época. Sin embargo, esto no ocurrió pacíficamente. Wolff encontró una fuerte oposición entre los teólogos pietistas de Halle, que gozaban de gran prestigio junto al entonces Rey de Prusia, Federico Guillermo I. Tras sufrir sucesivas acusaciones de ateísmo y determinismo por parte de los pietistas, Wolff fue expulsado del territorio prusiano en 1723 , y luego se vio obligado a exiliarse en Marburg, donde ocupó una cátedra de profesor durante los siguientes 17 años. Fue en este período que inició la presentación de su sistema en lengua latina, incluyendo sus dos tratados de psicología-Psychologia empirica (1732) y Psychologia rationalis (1734)-, y logrando así el reconocimiento internacional. En 1740, el rey sucesor de Prusia, Federico el Grande, le invitó a volver a Halle, donde fue recibido como un héroe (Araujo, 2012; Corr, 2003; Dreschsler, 1997; Hettche, 2009).

A pesar de que fue a través de sus obras latinas que Wolff se convirtió en un autor de difusión internacional, y su psicología, en particular, llegó a formar parte de la agenda intelectual europea, esto no disminuye la importancia de sus escritos alemanes. De hecho, fue a través de ellos que Wolff hizo algunas de sus contribuciones más significativas al pensamiento y a la cultura alemana. Sus manuales de matemática y filosofía establecieron un nuevo vocabulario para la lengua alemana (Blackall, 1959) y fueron adoptados como referencias oficiales para la enseñanza universitaria. De esta manera, sus ideas tuvieron una gran difusión en Alemania, permitiendo el surgimiento del "wolffianismo" como primera gran escuela de la filosofía alemana (Frängsmyr, 1975). Gracias a sus contribuciones, fue nombrado "educador de la nación alemana" (praeceptor Germania) y recibió el título de "Barón del Sacro Imperio Germánico” (Reichsfreiherr), otorgado por la primera vez a un profesor (Drechsler, 1997; Hettche 2009; Schwaiger, 2000).

Esta breve contextualización histórica nos permite comprender la importancia de la MA, a pesar del éxito posterior de las obras latinas. La MA se convirtió en un verdadero best seller. Entre 1720 y 1754, tuvo doce ediciones, ganó un suplemento con notas explicativas y adiciones (Notas a la Metafísica Alemana, 1724), y fue comentada por más de 400 estudiosos (Corr, 2003; González Ruiz, 2000). Entre los factores que justifican tal reputación están su estilo, su 
método de exposición y el carácter innovador de la mayoría de las disciplinas incluidas allí, entre ellas la psicología.

De hecho, la psicología tiene un valor significativo en el sistema de wolffiano. En primer lugar, en la medida en que, para Wolff, todo conocimiento debe partir de la experiencia y encontrar ahí su criterio de validez, muchas disciplinas filosóficas van a extraer sus primeros principios de la psicología. Según Wolff, "al conocimiento que alcanzamos al prestar atención a nuestras sensaciones y a las modificaciones del alma lo solemos llamar experiencia" (Wolff, 1751/2003, p. 181, §.325 - énfasis en el original) ${ }^{2}$. La Lógica, la Moral y la Política, por ejemplo, buscan sus principios en la psicología, en la medida en que esta revela la naturaleza y el funcionamiento de la facultad cognoscitiva y apetitiva del alma humana (Wolff, 1751/2003, §.191). Además, la psicología también ofrece una base para la teología natural, una vez que las nociones acerca de los atributos de Dios se forman a partir de la eliminación de las limitaciones de las nociones acerca de los atributos del alma humana (Wolff, 1751/2003, §§.928-1089). Del mismo modo, proporciona principios seguros para la religión, al demostrar la autoridad del alma y garantizar su libertad y su inmortalidad (Wolff, 1751/2003, p. 46). Estos ejemplos, aunque incompletos, son suficientes para mostrar la importancia de la psicología en el pensamiento de Wolff.

En este contexto, resulta significativo el hecho de que la MA es la primera presentación de la psicología de Wolff. Fue a partir de ahí que su concepción y su vocabulario psicológico penetraran en la cultura alemana. Por lo tanto, si es verdad, como afirman algunos autores (Bell, 2005; Smith, 1997; Vidal, 2006; Watkins, 1998), que a través de la obra de Wolff la psicología ganó un espacio sin precedentes en la academia y en la vida cultural alemana, debemos reconocer en ese proceso también el papel de la MA.

2 Como es habitual entre los comentadores de Wolff, las referencias a su obra serán hechas a través de los respectivos párrafos. Pero en las citas indicaremos también las páginas antes de los párrafos.

\section{La psicología racional en la Metafísica Alemana}

Como indica su título completo, la MA contiene pensamientos racionales acerca de Dios, el mundo y el alma del hombre, así como sobre todas las cosas en general. Tales temas se relacionan con cada una de las cuatro disciplinas que formaban la metafísica de Wolff: teología natural, cosmología general, psicología y ontología, respectivamente. Con respecto a los "pensamientos racionales", su racionalidad está ligada al método filosófico de Wolff. Basándose en los principios de contradicción y razón suficiente, su punto de partida son aquellos conocimientos que él considera los más fundamentales, para ir progresivamente alcanzando los más elevados, de manera que todo antecedente contiene el fundamento de su sucesor. En resumen, es la búsqueda de un conocimiento demostrativo basado en primeros principios (Sorell, Rogers, \& Kraye, 2010).

Las disciplinas con sus respectivos capítulos ganan, así, la siguiente secuencia: 1- "De cómo sabemos que somos y para qué nos sirve este conocimiento" (\$§.1-9, inicio de la psicología empírica); 2- "De los primeros principios de nuestro conocimiento y de todas las cosas en general" ( $\$ \S .10$ 190, Ontología); 3- "Del alma en general, de lo que efectivamente percibimos de ella" (\$§.191-539, psicología empírica); 4- "Del mundo" (\$§.540-726, cosmología general); 5- "De la esencia del alma y de un espíritu en general” (\$\$.727-927, psicología racional); 6- "De Dios" (\$§.928-1089, teología natural).

Como vemos, la psicología ocupa tres de los seis capítulos de la obra, pero no de manera continua. Es decir, la reflexión psicológica es interrumpida por la ontología y la cosmología. Sin embargo, eso se puede explicar por el método wolffiano. Wolff empieza su investigación con el hecho psicológico de que somos conscientes de nuestra existencia. Pero antes de proseguir con la psicología, Wolff tiene que esclarecer las bases lógicas de este y de todo conocimiento, así como las nociones generales que serán utilizadas para el conocimiento de todas las cosas, incluso del alma. Esto es llevado a cabo en su ontología. A continuación, después de la exposición de las facultades cognitivas y volitivas fundamentales 
del alma, y su sintonía con el cuerpo, la explicación de los estados psicológicos viene a depender de las nociones de cuerpo y mundo, que son proporcionadas por la cosmología. Solo entonces, se puede volver a la psicología para completarla.

Teniendo esto en cuenta, es necesario esclarecer dos aspectos de nuestro análisis. Primero, es importante decir que Wolff todavía no utiliza los nombres psicología racional y psicología empírica para separar las dos partes de su psicología en la MA. Estos términos solo van a aparecer por primera vez en la introducción a los tratados latinos, publicada inicialmente en 1728 (Wolff, 1740/1983a, §§. 111112). Sin embargo, dado que los temas son idénticos, vamos a adoptar aquí la terminología clásica también para la MA. En segundo lugar, puesto que algunas explicaciones psicológicas dependen de conocimientos de otras disciplinas, como la ontología y la cosmología, vamos a incluirlos asimismo, siguiendo el orden de presentación de Wolff.

\section{Psicología racional en general}

Cuatro temas cubren los contenidos de la psicología racional: la esencia del alma, la relación cuerpoalma, la fundamentación de las facultades del alma y el conocimiento sobre los espíritus en general. En el pasaje en que introduce la psicología racional (PR), Wolff dice:

Anteriormente, en el capítulo tercero, he estudiado ya por extenso el alma, pero sólo en la medida en que percibimos sus efectos y podemos alcanzar un concepto distinto de ellos (\$.191). Ahora hemos de investigar en qué consiste la esencia del alma y de un espíritu en general, y cómo se fundamenta en esta esencia lo que percibimos de ella y hemos indicado anteriormente. En lo cual, sin embargo, se podrán tratar respecto del alma diferentes aspectos a los cuales no nos conduce la experiencia de modo inmediato. (Wolff, 1751/2003, p. 453, §.727)

En este pasaje, vemos que la PR representa una profundización del conocimiento sobre el alma, complementando el cuadro ya proporcionado por la psicología empírica ${ }^{3}$. Sin embargo, al mismo tiempo ella contiene los fundamentos de todo lo que fue aprendido en esta última. Por lo tanto, la PR tiene un papel tanto de expansión como de justificación del conocimiento psicológico. Como vimos en la sección anterior, sin embargo, para realizar esta función ella debe tener en cuenta no solo el conocimiento empírico de la psicología, sino también otras nociones metafísicas. Vamos a empezar por estas últimas.

\section{Sobre las cosas en general}

El primer conocimiento fundamental del cual depende la psicología racional se encuentra en la ontología: la determinación de la noción de cosa simple (\$§. 75-80). Wolff sugiere que, inicialmente, notemos que todas las cosas que conocemos por la experiencia poseen partes y son, por eso, llamadas de cosas compuestas. Entre ellas se encuentran, por ejemplo, todos los cuerpos y cosas materiales que, cuando son tomados en su totalidad, constituyen el mundo. Si aceptamos al principio de razón suficiente, según el cual todo tiene una razón de ser, debemos también admitir, a pesar de nunca atestiguarla en la experiencia, la existencia de algo que da origen a las cosas compuestas y que, por su vez, no puede ser el mismo un compuesto, pues en este caso el resultado sería la divisibilidad infinita de las partes. Hay, por consiguiente, cosas sin partes, a las cuales Wolff da el nombre de cosas simples.

Todas las cosas (compuestas o simples), continúa Wolff, poseen algo inmutable por lo cual son como son y no de otra forma, es decir, contienen la razón por la cual todos sus aspectos les corresponden. Esto se llama esencia, y sus aspectos, propiedades (§§. 32-44). Los límites de la esencia, no obstante, pueden variar (como en el caso de la alteración de la cera de una vela, sin que esta deje de ser lo que es). Tales variaciones son los cambios, mientras que las limitaciones resultantes son los estados. La razón de todo cambio es la fuerza de las cosas, que

3 Vimos en la sección anterior que el contenido de la psicología empírica fue tratado en el capítulo tercero - "Del alma en general, de lo que efectivamente percibimos de ella" (\$§.191-539). 
consiste en el afán de la cosa de cambiar sus límites. Cuando la fuerza pertenece a la propia cosa que cambia, el cambio es una acción, y la cosa es existente por símisma, o sea, una sustancia. Cuando pertenece a otra cosa, aquella que cambia es una simple limitación de una sustancia, y su cambio es una pasión (§§.107-117).

La esencia de las cosas compuestas consiste en su estructura, o sea, en la organización (composición) de sus partes. Así, sabemos que se originan a través de composición y dejan de existir por descomposición de las partes, y tienen como propiedad la extensión, la magnitud, la forma, la ocupación del espacio y el movimiento (\$§. 51-73). Los cuerpos, bajo la condición de cosas compuestas, se definen por ser compuestos de materia y poseer fuerza motriz ( $\$ .626$ en la cosmología general).

Las cosas simples, al contrario, no poseen ninguna de estas propiedades, y por eso no se originan por composición y no dejan de existir por descomposición (\$§. 82-91). Hay en ellas algo perenne que, no obstante, tiene límites mutables, de los cuales derivan las diferencias en las cosas compuestas. Como ni reciben ni dan nada, tales variaciones deben fundamentarse en ellas mismas, y constituyen, pues, acciones. Puede inferirse así que son sustancias y poseen una fuerza, que es su esencia y mediante la cual modifican de manera continua y autónoma su estado (§§. 112-127). Aquí, Wolff ofrece un ejemplo significativo:

Por ejemplo, nuestra alma posee una fuerza mediante la cual produce sus pensamientos sucesivamente en estricto orden, y es por ello una cosa existente por sí misma. En cambio, tanto los conceptos por ella producidos como su apetito (...) no son más que limitaciones de esa fuerza. (Wolff, 1751/2003, pp. $59-60, \S .114)$

Así, queda claro que para Wolff el alma consiste en una cosa simple y que tiene una fuerza a través de la cual produce de modo constante todos sus efectos. Podemos ahora volver a la psicología y avanzar en el conocimiento racional del alma.

\section{De la esencia del alma}

La investigación sobre la esencia del alma empieza con el primer conocimiento de la psicología empírica: a) somos conscientes de nosotros y de otras cosas fuera de nosotros; b) el alma es aquella cosa en nosotros que es consciente de sí misma y de otras cosas fuera de ella (\$§. 728-734). Esta consciencia tiene su origen en la percepción de la diferencia entre nosotros y las otras cosas. O sea, no solo la representación, sino también la comparación y diferenciación de las cosas son necesarias para el surgimiento de la consciencia. De ahí, Wolff llega a una definición negativa: el alma no es material (§§. 738-741). Los cuerpos representan objetos mediante la disposición que sus estructuras asumen frente a ellos, pero no comparan tal representación con el objeto fuera de ellos y, por lo tanto, no poseen consciencia.

Como el alma no puede ser compuesta de materia, dice Wolff, tiene que ser una cosa simple $(\$ \S$. 742-752). Luego, posee una fuerza de la cual surgen todos sus cambios y en la cual consiste su esencia. Así, se comprende que todas sus facultades no son más que diferentes limitaciones de una misma fuerza, a las cuales se dan diferentes nombres. Esta fuerza fundamental, Wolff concluye, es la de "representarse el mundo según la posición de su cuerpo en el mismo" (Wolff, 1751/2003, p. 468, §.753).

El próximo paso es comprender cómo puede el alma representar el mundo. Para solucionar eso, Wolff investiga la sintonía entre el cuerpo y el alma.

\section{La sintonía entre cuerpo y alma}

Wolff admite que la psicología empírica solamente nos garantiza la existencia de la sintonía entre cuerpo y alma, pero no puede darnos su razón (§§. 527-539). No obstante, la experiencia mantiene una función esencial: sirve de criterio de evaluación para el análisis de teorías. O sea, las teorías más probables son aquellas que más se adecuan a los datos de la experiencia. En el Prefacio a la primera edición de la MA, Wolff anuncia que, entre todas las teorías sobre la relación cuerpo-alma, solo hay una que atiende a este criterio: 
Ciertamente me había propuesto al principio dejar sin respuesta alguna la cuestión de la comunidad alma-cuerpo: sin embargo, dado que me vi conducido inesperadamente y de un modo completamente natural [...] a la armonía preestablecida del señor von Leibniz, también yo la he mantenido y he proyectado sobre ella más luz de la que poseyera nunca esta ingeniosa creación. (Wolff, 1751/2003, p. vii - énfasis en el original)

Para Wolff, debemos aceptar el hecho de que tanto el alma como el cuerpo poseen sus propias fuerzas, mediante las cuales producen sus cambios de manera autónoma, sucesiva y constante. En otras palabras, uno no necesita del otro. Luego, tiene que haber una razón para la sintonía observada en la experiencia. Según él, si las sensaciones del alma representan los cambios del mundo, es posible que estas dos cadenas de cambios fueran armonizadas por Dios en el acto de la creación, para que se mantuvieran armonizadas naturalmente para siempre. En esto consiste la armonía preestablecida. Y puesto que ella no contradice ni la experiencia ni las leyes ya demostradas en su sistema filosófico, es aceptada por Wolff.

Sin embargo, Wolff se da cuenta de dos dificultades de esta teoría ( $\$$. 781). La primera consiste en la demonstración de cómo es posible que el cuerpo, mediante sus movimientos y sin ninguna contribución del alma, actúe racionalmente, expresando verdades universales, haciendo inferencias y descubrimientos, como nos enseña la experiencia. La segunda es la demonstración de la libertad del hombre, ya que el cuerpo no posee entendimiento para hacer elecciones y tiene todos sus movimientos determinados necesariamente por los movimientos de otros cuerpos, que a su vez tampoco lo tienen.

De modo general, vemos que la primera dificultad está relacionada con la dimensión cognitiva, mientras que la segunda a la dimensión volitiva del hombre. Para solucionarlas, Wolff analiza como las actividades del alma se fundamentan en su fuerza representativa ( $\$$. 782-885). En el segundo Prefacio a la MA, afirma: "todo lo que se deduce en el capítulo quinto sobre los atributos y actos del alma a partir de su naturaleza, no fuera otra cosa que una explicación de la armonía preestablecida entre alma y cuerpo" (Wolff, 1751/2003, p. viii). Wolff alcanza, así, dos objetivos de su psicología racional: demostrar cómo se dan las actividades del alma (solamente) a partir de su fuerza fundamental y cómo es posible la armonía preestablecida.

\section{Las actividades del alma}

Wolff empieza su demonstración con la sensación, la actividad más elementar del alma (\$§. 783-819). La pregunta aquí planteada es la siguiente: ien qué sentido se puede decir que las sensaciones se fundamentan en la fuerza representativa, si ellas son cambios del alma derivadas de los cambios que los objetos producen en nuestro cuerpo? Primeramente, partiendo de la definición de fuerza representativa, Wolff destaca que esta posee una limitación: la posición del cuerpo en el mundo. $\mathrm{O}$ sea, a pesar de abarcar el mundo en su totalidad, el alma representa sucesivamente sus partes, según el lugar del cuerpo. Las sensaciones, así, poseen el mismo fundamento que los cambios en nuestro cuerpo y en el mundo, y parecen, pues, consistir en pasiones. Sin embargo, afirma Wolff, todo lo que el alma puede representar ya está dado como posibilidad en su esencia, y por su propia fuerza es sucesivamente actualizado, lo que ocurre en sintonía con el cuerpo. Las sensaciones permanecen, así, acciones del alma, y tienen razón en su fuerza.

Una vez comprendido cómo las sensaciones surgen de la fuerza representativa, las facultades siguientes se derivan con facilidad (\$§. 807-847). La imaginación, que es la facultad que produce representaciones de cosas ausentes debido a su semejanza con sensaciones presentes, y la memoria, la facultad de reconocer una representación como ya representada, no son otra cosa que variaciones de la fuerza representativa. Lo mismo acontece con la atención y la reflexión, que se refieren a la capacidad del alma de incrementar la consciencia sobre una representación. Y también con las demás capacidades cognitivas: el entendimiento y sus elementos (juicio, conocimiento simbólico, inferencias, ciencia), la capacidad de representar con distinción (determinando las diferencias entre 
las cosas), y la razón, la capacidad de representar la interconexión de las representaciones.

Debido a la sintonía del cuerpo con el alma, todas las acciones de la última son acompañadas en lo primero por movimientos especiales de la materia cerebral (llamados representaciones materiales). Así, Wolff tiene lo suficiente para solucionar la primera dificultad relacionada a la armonía preestablecida (\$§. 835-845). Como las palabras consisten en sonidos, dice él, y actúan como signos del conocimiento, este es representado en el cuerpo a partir de los movimientos que ellas provocan en los oídos y en el cerebro. Es decir, ocurren movimientos en el cuerpo con los cuales se sintoniza el conocimiento del alma. Y una vez asociados los movimientos causados por las palabras con aquellos provocados por los objetos reales designados por las palabras, cuando estos vuelven a acontecer, los primeros también suceden. Así, el cuerpo puede producir, como una simple máquina, las palabras e inferencias que se adecuan al objeto y al conocimiento del alma, es decir, puede actuar racionalmente sin participación del alma. Sin embargo, debido a la ausencia de consciencia, sigue siendo la razón una propiedad exclusiva del alma.

Volviendo a las actividades volitivas del alma (§§. 873-883), se nota que Wolff intenta responder a otra cuestión: ien qué sentido el placer, el deseo y la voluntad se originan en la fuerza representativa? Retomando los conceptos en cuestión, Wolff concibe el placer como la representación de la perfección de una cosa, y el displacer como la representación de la imperfección. Además, él da el nombre de "bien" a lo que perfecciona nuestro estado, y de "mal" a lo que nos trae imperfección. Así, afirma que la volición sigue una regla según la cual nosotros nos inclinamos a lo que tenemos por bien y rechazamos a lo que tenemos por mal. A estos fenómenos él da el nombre de deseo o aversión, cuando la representación del bien o del mal es indistinta; y de voluntad y no querer, cuando ella es distinta. Por último, para aclarar como la inclinación o el rechazo pueden derivar de la misma fuerza representativa, Wolff explica que esta última no consiste solo en la actividad de representar, sino también en la de modificar constantemente las representaciones. Tales cambios son las inclinaciones y los rechazos del alma.
Después de haber visto como el placer, el deseo y la voluntad surgen de la fuerza representativa del alma, Wolff afirma que la libertad -la facultad de elegir espontáneamente entre dos cosas igualmente posibles la que ella representa como más agradable- debe también originarse de su fuerza representativa (\$§. 883-885). Así, por determinar a sí misma sin ninguna contribución del cuerpo, el alma se encuentra libre de la coacción externa; pero también de la interna, pues procede de acuerdo con el entendimiento, y no por necesidad. Esto significa que, con la armonía, el alma posee la mayor libertad que se puede pensar.

En cuanto a la segunda dificultad de la armonía preestablecida -la libertad del hombre-, dice Wolff, se debe reconocer que, dada la sintonía original entre cuerpo y alma, no hay diferencia entre imaginar que el alma determine el cuerpo para el movimiento, o que el curso de la naturaleza esté dispuesto de tal forma que los seres corporales obligan al cuerpo a moverse de acuerdo con la voluntad del alma. Wolff admite que, no obstante, no sería imposible que el curso de la naturaleza y la voluntad de los hombres vieran determinados a perjudicar el deseo del alma. Sin embargo, ya que tampoco es imposible que se sintonicen, debemos aceptar definitivamente como posible la armonía preestablecida.

Wolff concluye, así, los objetivos de demostrar el fundamento de las actividades del alma y de su relación con el cuerpo. No obstante, hay todavía una última cuestión por resolver.

\section{El concepto de espíritu en general}

Wolff contempla aún en su psicología racional una exposición sobre los espíritus (§§. 788-927). En su inicio (§§. 788-794), admite simplemente que es posible que todo cuerpo dotado de órganos sensoriales tenga un alma. Así, para él, es concebible que también los animales, que poseen cuerpos con órganos sensoriales, tengan almas que representen el mundo según su posición en él y con facultades semejantes a las humanas. Estas almas, sin embargo, ya que son limitadas por cuerpos inferiores a los de los hombres, son también inferiores a las nuestras. Comparándolas con las almas de los hombres (§§. 
869-891), Wolff concluye que ellas difieren sobre todo por la ausencia de entendimiento y por su consecuente irracionalidad y esclavitud (el contrario de la libertad).

El próximo paso es el reconocimiento de que, dadas las diferentes calidades de representación posibles para una fuerza representativa, hay aún otros seres semejantes al alma humana, a los cuales todavía no se han dado nombres (§§. 899-903). Su primera especie es constituida por seres que representan el mundo obscuramente y nada pueden discernir, de forma que no poseen consciencia. En la segunda, se encuentran cosas como las almas de los animales, que representan clara e indistintamente, es decir, perciben las diferencias entre las cosas y son conscientes, pero no pueden comprenderlas. En la tercera, finalmente, están aquellas cosas que pueden representar clara y distintamente, siendo denominadas espíritus, entre los cuales hay muchas subespecies con diferentes grados de limitación, como las almas, que consisten en espíritus limitados por la posición del cuerpo en el mundo.

Reconocido el cuadro de las cosas semejantes al alma, Wolff expone sus últimas propiedades (§§. 904-927). Como ya había demostrado en la ontología, porque son cosas simples, su existencia no cesa por descomposición, como a la de los compuestos. Tal condición es lo que Wolff llama de incorruptibilidad. Los espíritus, sin embargo, porque poseen aún entendimiento y voluntad libre, desarrollan también sabiduría -la ciencia de elegir los medios (más breves) para los fines-y personalidad, la capacidad de comparar sus estados y tener consciencia de que siguen siendo los mismos. Por eso, además de la incorruptibilidad, los espíritus poseen también inmortalidad, que consiste en la conservación del estado de la personalidad incluso después de la muerte.

Con eso, Wolff concluye su exposición sobre el alma humana y los espíritus en general y también su psicología racional. La MA sigue, no obstante, con la investigación teológica sobre las propiedades de Dios (\$§.928-1089), que en el sistema de Wolff también es un ser espiritual. De hecho, la psicología racional desempeña todavía otros papeles en el sistema wolffiano, que no es posible tratar aquí.

\section{Consideraciones finales}

La psicología racional ocupa un lugar central en el sistema wolffiano, proveyendo fundamentos a la psicología empírica y principios a la teología natural. Sin embargo, su significado para el pensamiento de Wolff va mucho más allá. Debemos tener en cuenta aquí, una vez más, el papel de Wolff como Aufklärer. En este contexto, la psicología racional es parte esencial del proyecto wolffiano de fundamentación de conocimientos todavía fuertemente asociados a la tradición religiosa. La inmortalidad y el libre arbitrio del alma humana, la fundamentación de la espiritualidad y el esclarecimiento de la relación entre cuerpo y alma constituyen sus mayores ejemplos. Como dijimos antes, Wolff dedicó gran parte de sus esfuerzos a la defensa de su sistema y de la libertad de pensamiento en general, lo cual llegó a ser una marca de su pensamiento, aún antes de la publicación de sus escritos latinos.

De hecho, este papel central de la psicología racional, no solo en el sistema de Wolff sino también en su vida personal, es evidente en las Notas a la Metafísica Alemana (Wolff, 1740/1983b, pp. 122127, §.55), cuando él revela todo su esfuerzo para librarse de las acusaciones políticas y religiosas resultantes de su defensa de la doctrina de la armonía preestablecida, que produjo su expulsión de Halle, como vimos anteriormente.

Además, los problemas que tuvo que enfrentar Wolff por el contenido de su psicología racional en la MA parecen constituir una de las razones de su propuesta de una separación más nítida con respecto a la psicología empírica, como revela el siguiente pasaje del Discurso Preliminar:

En la psicología racional derivamos a priori, a par-
tir de un único concepto del alma humana, todas
las cosas que son posteriormente observadas como
pertenecientes al alma y todas las cosas que son de-
ducidas de estas observaciones, en la medida que son
propias de la filosofía. Este es un nuevo y audaz em-
prendimiento que es contrario a la opinión anterior.
La mayoría de las personas suelen ser inicialmente
reacias a admitir cosas nuevas. La razón principal
por la que distinguí entre psicología racional y em- 
pírica fue impedir que el conocimiento psicológico fuera rechazado indiscriminadamente. Los principios psicológicos son necesarios para la teoría y la práctica de la moral y la política, así como para las cosas que deducimos de ellas. La filosofía práctica es de la mayor importancia, y no queremos que se base en principios cuestionables. (Wolff, 1740/1983a, p. $51, \S .112)$

Queda claro así que la continuación entre la psicología racional de la MA y la de los tratados latinos es un tópico que, muy lejos de ser trivial o irrelevante, puede esclarecer aspectos importantes del desarrollo y de la sistematización del pensamiento wolffiano. A la vez, muestra la necesidad de investigaciones futuras más detalladas de la MA.

Pero no es solo en el interior del pensamiento de Wolff que la psicología racional tuvo un papel importante. Redefiniendo y consolidando nuevas fronteras en el estudio del alma, hasta entonces diluidas entre varios proyectos distintos (pneumatología, moral, religión, etc.), Wolff posibilitó el surgimiento de una tradición alemana de debates sobre la psicología en el siglo XVIII (Watkins, 1998). Filósofos como A. Baumgarten (1714-1762) y M. Mendelssohn (1729-1786), entre muchos otros, fueran actores importantes de esta tradición, continuando en muchos aspectos el proyecto wolffiano de una psicología racional o metafísica. Además, fue esa misma tradición que sirvió de base a la clásica crítica de I. Kant (1724-1804) a las nociones de sustancialidad, personalidad e inmortalidad del alma (Kant, 1781/1998). O sea, la idea wolffiana de una psicología racional tuvo en el siglo XVIII una recepción tanto positiva como negativa. En ambos casos, sin embargo, ella sirvió como base para las discusiones psicológicas y metafísicas.

En el siglo XIX, esa doble tendencia se mantuvo. Por un lado, J. F. Herbart (1776-1841), aunque no propuso una separación en los moldes wolffianos, defiendió una psicología metafísica con muchas influencias de la tradición leibniz-wolffiana (Herbart, 1850). Por el otro, en el momento en que muchos psicólogos procuraban distanciarse cada vez más de las especulaciones metafísicas sobre la naturaleza del alma, la distinción establecida por Wolff entre psicología empírica y racional aún era tomada como base para muchas discusiones. El joven Wundt, por ejemplo, en su primer texto teórico sobre la situación de la psicología, se refirió explícitamente a Wolff, afirmando que la psicología racional debería ser abandonada para que la psicología empírica pudiera ser desarrollada con completa autonomía (Wundt, 1862, pp. xi-xxxii).

Pero Wundt es solo un ejemplo. En efecto, algunos de los problemas planteados por Wolff en su psicología racional no solo formaron parte de la agenda de muchos otros psicólogos importantes, como William James (Brown, 2008), sino que también continúan presentes en muchos debates interdisciplinares contemporáneos (Viger, Bluhm, \& Mosurinjohn, 2008). Sin embargo, esta también es una cuestión que merece ser tratada en investigaciones futuras, y para la cual solo pudimos ofrecer aquí algunas indicaciones. No obstante, esperamos haber mostrado que hay razones suficientes para dar a Wolff un lugar más destacado en la historia de la psicología.

\section{Referencias}

Araujo, S. F. (2012). O lugar de Christian Wolff na história da psicologia. Universitas Psychologica, 11(3), 1013-1024.

Baldwin, J. M. (1913). History of psychology: An sketch and an interpretation (Vols. 1-2). New York: The Knickerbocker Press.

Bell, M. (2005). The German tradition of psychology in literature and thought, 1700-1840. Cambridge: Cambridge University Press.

Blackall, E. (1959). The emergency of German as a literary language. Cambridge: Cambridge University Press.

Blackwell, R. J. (1961). Christian Wolff's doctrine of the soul. Journal of the History of Ideas, 22(3), 339-354.

Boring, E. G. (1950). A history of experimental psychology. New York: Appleton-Century-Crofts.

Bossenbrook, W. (1961). The German mind. Detroit: Wayne State University Press.

Brett, G. S. (1921). A history of psychology (Vol. 2). London: George Allen \& Unwin.

Brown, H. (2008). William James and the Achilles argument. In T. Lennon \& R. Stainton (Eds.), The 
Achilles of rationalist psychology (pp. 247-255). New York: Springer.

Corr, C. A. (1975). Christian Wolff's distinction between empirical and rational psychology. Studia Leibnitiana Supplementa, 14, 195-215.

Corr, C. A. (2003). [Introduction]. En J. École (Ed.), Christian Wolffs Gesammelte Werke (I, Vol. 2.1, pp. 1-47). Hildesheim: Olms.

Drechsler, W. (1997). Christian Wolff: A Biographical Essay. European Journal of Law and Economics, 4, 111-128.

École, J. (1969). De la nature de l'âme, de la déduction de ses facultés, de ses rapports avec le corps, ou la 'Psychologia rationalis' de Christian Wolff. [De la naturaleza del alma, de la dedución de sus facultades, de su relación con el cuerpo, o la 'Psychologia rationalis' de Christian Wolff]. Giornale di Metafisica, 24, 499-531.

École, J. (2001). Christian Wolff était-il un Aufklärer? [¿Fué Christian Wolff un Aufklärer?] En J. École (Ed.), Christian Wolffs Gesammelte Werke (III, Vol. 65, pp. 172-185). Hildesheim: Olms.

Feuerhahn, W. (2002). Comment la psychologie empirique est-elle née? [¿Como nació la psicología empírica?] Archives de Philosophie, 65(1), 47-64.

Frängsmyr, T. (1975). Christian Wolff's mathematical method and its impact on the eighteenth century. Journal of the History of Ideas, 36(2), 653-668.

González Ruiz, A. (Ed.). (2000). [Presentación]. En C. Wolff, Pensamientos racionales acerca de Dios, el mundo y el alma del hombre, así como sobre todas las cosas en general: (Metafísica Alemana) (pp. 7-31). Madrid: Akal.

Goodwin, C. J. (2005). História da psicologia moderna (M. Rosas, Trad., 2a. ed.). São Paulo: Cultrix.

Gundlach, H. (2006). Psychology as science and as discipline: The case of Germany. Physis. Rivista Internazionale di Storia della Scienza, 18, 61-89.

Hatfield, G. (1995). Remaking the science of mind: Psychology as natural science. En C. Fox, R. Porter \& R. Wokler (Eds.), Inventing human sciences: Eighteenth-century domains (pp. 184-231). Berkley: University of California Press.

Herbart, J. (1850). Psychologie als Wissenschaft, neu gegründet aufErfahrung, Metaphysik und Mathematik. [Psicologia como ciencia, recién fundada sobre la experiencia, la metafísica y la matemática]. En G. Hartenstein (Ed.), Herbarts Sämmtliche Werke (Vol. 5). Leipzig: Voss.

Hettche, M. (2009). Christian Wolff. En E. Zalta (Ed.), The Stanford encyclopedia of philosophy. Available at http://plato.stanford.edu/archives/spr2009/entries/ wolff-christian/

Hothersall, D. (2006). História da psicologia. São Paulo: McGraw-Hill.

Kant, I. (1998). Kritik der reinen Vernunft. [Crítica de la razón pura]. Hamburg: Meiner. (Trabajo original publicado en 1781)

Marcolungo, F. L. (Ed.). (2007). Christian Wolff tra psicologia empirica e psicologia razionale. Atti del seminario internazionale di studi, Verona, 13-14 maggio, 2005. En Christian Wolff: Gesammelte Werke (Bd. 106, Wolffiana 3). Hildesheim: Olms.

Mei, M. (2011). L'intuizione dei pensieri. La pars inferior animae nella psicologia cognitiva di Christian Wolff. Roma: Aracne.

Molina, J. (2010). Christian Wolff y la psicología de la ilustración alemana. Persona, 13, 125-136.

Pütz, P. (1978). Die deutsche Aufklärung. [La Ilustración Alemana]. Darmstadt: Wissenschaftliche Buchgesellschaft.

Robinson, D. (1995). An intellectual history of psychology (3a. ed.). Madison, Wisconsin: University of Wisconsin Press.

Rudolph, O. -P., \& Goubet, J. -F. (Eds.). (2004). Die Psychologie Christian Wolffs. Systematische und historische Untersuchungen. [La psicología de Christian Wolff. Investigaciones sistemáticas e históricas]. Tubingen: Niemeyer.

Schultz, D., \& Schultz, S. (2011). A history of modern psychology (10a. ed.). Belmont, CA: Cengage Learning.

Schwaiger, C. (2000). Christian Wolff: A figura central do iluminismo alemão. em L. Kreimendahl (Ed.), Filósofos do século XVIII: uma introdução (pp. 65. 88). São Leopoldo, RS: Unisinos.

Smith, R. (1997). The Norton History of the Human Sciences. New York: W. W. Norton \& Company.

Sorell, T., Rogers, G. A. J., \& Kraye, J. (2010). Scientia in early modern philosophy. New York: Springer.

Sturm, T. (2006). Is there a problem with mathematical psychology in the eighteenth century? A fresh look 
on Kant's old argument. Journal of the History of the Behavioral Sciences, 42(4), 353-377.

Vidal, F. (2006). Les sciences de l'âme: XVI éme_XVIII éme siècle. [Las ciencias del alma: siglos XVI - XVIII]. Paris: Honoré Champion.

Viger, C., Bluhm, R., \& Mosurinjohn, S. (2008). The binding problem: Achilles in the 21st century. En T. Lennon \& R. Stainton (Eds.), The Achilles of rationalist psychology (pp. 257-275). New York: Springer.

Watson, R. I. (1963). The great psychologists. Philadelphia: Lippincott.

Watkins, E. (1998). From pre-established harmony to physical influx: Leibniz's reception in eighteenth century Germany. Perspectives on Science, 6(1-2), 136-203.

Wolff, C. (1983a). Philosophia rationalis sive logica (Pars 1). [Filosofía racional o lógica (Parte 1)]. En C. Corr (Ed.), Christian Wolffs Gesammelte Werke
(II, Vol. 1). Hildesheim: Olms. (Trabajo original publicado en 1740).

Wolff, C. (1983b). Anmerkungen zur Deuteschen Metaphysik. [Notas a la Metafísica Alemana]. En C. Corr (Ed.), Christian Wolffs Gesammelte Werke (I, Vol. 3). Hildesheim: Olms. (Trabajo original publicado en 1740).

Wolff, C. (2003). Vernünftige Gedanken von Gott, der Welt und der Seele des Menschen, auch allen dingen überhaupt. [Pensamientos racionales acerca de Dios, el mundo y el alma del hombre, así como sobre todas las cosas en general]. En C. Corr (Ed.), Christian Wolffs Gesammelte Werke (I, Vol. 2). Hildesheim: Olms. (Trabajo original publicado en 1751).

Wundt, W. (1862). Beiträge zur Theorie der Sinneswahrnehmungen. [Pensamientos racionales acerca de Dios, el mundo y el alma del hombre, así como sobre todas las cosas en general]. Leipzig: Winter. 